\title{
ON CALABI-YAU THREEFOLDS WITH LARGE NONABELIAN FUNDAMENTAL GROUPS
}

\author{
LEV BORISOV AND ZHENG HUA \\ (Communicated by Ted Chinburg)
}

\begin{abstract}
In this short note we construct Calabi-Yau threefolds with nonabelian fundamental groups of order 64 as quotients of the small resolutions of certain complete intersections of quadrics in $\mathbb{P}^{7}$ that were first considered by M. Gross and S. Popescu.
\end{abstract}

\section{INTRODUCTION}

In [GPo], M. Gross and S. Popescu constructed Calabi-Yau varieties that admit a free action of the group $(\mathbb{Z} / 8 \mathbb{Z})^{2}$. We observe that these varieties admit a larger automorphism group, and that two other subgroups of order 64 of this group act freely. Our construction is very simple, modulo the nontrivial calculations of [GPo]. The essential idea is that in the presence of only two nonisomorphic, minimal models every automorphism of the singular variety $V_{8, y}$ naturally extends to the models. We then construct the additional automorphisms explicitly as permutations of coordinates.

In the last section we very briefly describe what led to the discovery of these examples and how they fit into a more general question of finding free group actions on complete intersections of quadrics.

\section{Construction}

We recall the definition of the Calabi-Yau varieties of Gross and Popescu, [GPo, $\mathrm{GPa}$. Consider the projective space $\mathbb{C P}^{7}$ with coordinates $\left(x_{0}: \ldots: x_{7}\right)$. For any $\left(y_{0}: y_{1}: y_{2}\right) \in \mathbb{C P}^{2}$ in a general position, consider the intersection $V_{8, y}$ of four quadrics

$$
\begin{aligned}
& y_{1} y_{3}\left(x_{0}^{2}+x_{4}^{2}\right)-y_{2}^{2}\left(x_{1} x_{7}+x_{3} x_{5}\right)+\left(y_{1}^{2}+y_{3}^{2}\right) x_{2} x_{6}=0 \\
& y_{1} y_{3}\left(x_{1}^{2}+x_{5}^{2}\right)-y_{2}^{2}\left(x_{2} x_{0}+x_{4} x_{6}\right)+\left(y_{1}^{2}+y_{3}^{2}\right) x_{3} x_{7}=0 \\
& y_{1} y_{3}\left(x_{2}^{2}+x_{6}^{2}\right)-y_{2}^{2}\left(x_{3} x_{1}+x_{5} x_{7}\right)+\left(y_{1}^{2}+y_{3}^{2}\right) x_{4} x_{0}=0 \\
& y_{1} y_{3}\left(x_{3}^{2}+x_{7}^{2}\right)-y_{2}^{2}\left(x_{4} x_{2}+x_{6} x_{0}\right)+\left(y_{1}^{2}+y_{3}^{2}\right) x_{5} x_{1}=0
\end{aligned}
$$

This variety has 64 ODP singular points which are the orbit of $\left(0: y_{1}: y_{2}: y_{3}: 0\right.$ : $\left.-y_{3}:-y_{2}:-y_{1}\right)$ under the action of the group $G=(\mathbb{Z} / 8 \mathbb{Z})^{2}$ generated by $\tau$ and

Received by the editors October 16, 2006 and, in revised form, February 17, 2007.

2000 Mathematics Subject Classification. Primary 14J32.

The first author was partially supported by the National Science Foundation under grant No. DMS-0456801. 
$\sigma$ where $\tau\left(x_{i}\right)=\zeta_{8}^{-i} x_{i}$ and $\sigma$ is a cyclic permutation of the variables given by the cycle (01234567). This group $G$ acts freely on $V_{8, y}$.

The variety $V_{8, y}$ admits two small resolutions, $V_{8, y}^{1}$ and $V_{8, y}^{2}$, both of which are Calabi-Yau threefolds with $h^{1,1}=h^{1,2}=2$. These resolutions are related by a flop and are obtained from $X$ by blowups of abelian surfaces in $X$ of degrees 32 and 16, respectively.

The key to the current note is the following lemma that relies heavily on the results of $[\mathrm{GPo}]$.

Lemma 2.1. Every automorphism $\gamma$ of $V_{8, y}$ lifts to automorphisms of $V_{8, y}^{1}$ and $V_{8, y}^{2}$. If $\gamma$ acts freely on $V_{8, y}$, then its lifts act freely on the small resolutions.

Proof. We will show that $\gamma$ lifts to $V_{8, y}^{2}$, with the other case being completely analogous.

As in [GPo], we can think of $V_{8, y}^{2}$ as the blowup of $V_{8, y}$ by the ideal of some Weil divisor $A$. Then there is a natural isomorphism $\hat{\gamma}$ from $V_{8, y}^{2}$ to the blowup of $V_{8, y}$ by the ideal of $\gamma(A)$. Denote the latter blowup by $Z$. It is clearly a minimal model of $V_{8, y}$, so by the observation of [GPo], $Z$ is isomorphic to either $V_{8, y}^{2}$ or $V_{8, y}^{1}$ (over $V_{8, y}$ ). To prove the first assertion of the lemma, it remains to show that $V_{8, y}^{2}$ and $V_{8, y}^{1}$ are not isomorphic, since we could then compose $\hat{\gamma}$ with an isomorphism $Z \rightarrow V_{8, y}^{2}$ over $V_{8, y}$. From the description of their Kähler cones we can see that any such isomorphism between $V_{8, y}^{i}$ would have to respect their structures as fibrations over $\mathbb{P}^{1}$ as well as their maps to $\mathbb{P}^{7}$. However, the degrees of the fibers are different in these two cases, hence $V_{8, y}^{2} \nRightarrow V_{8, y}^{1}$.

To show the last assertion, observe that if the lift of $\gamma$ had a fixed point, then the image of that point in $V_{8, y}$ would be fixed by $\gamma$.

We can now describe our construction.

Proposition 2.2. We define the group $G_{1}$ generated by $\tau$ and the permutation $\sigma_{1}=(07214365)$ of the coordinates $x_{i}$. Then $G_{1}$ is a nonabelian group isomorphic to a semidirect product of two copies of $\mathbb{Z} / 8 \mathbb{Z}$. We define the group $G_{2}$ generated by $\tau$ and the permutations $\sigma_{2}=(0246)(1357)$ and $\sigma_{3}=(0145)(3276)$. Then $G_{2}$ is a nonabelian group isomorphic to a semidirect product of the normal subgroup $\mathbb{Z} / 8 \mathbb{Z}$ generated by $\tau$ and the quaternion group $H$ generated by $\sigma_{2}$ and $\sigma_{3}$. Both $G_{1}$ and $G_{2}$ act freely on $V_{8, y}$.

Proof. The structure of $G_{1}$ and $G_{2}$ is immediate from their definition, and it is straightforward to see that $\sigma_{i}$ acts on $V_{8, y}$. To show that they act freely, it is enough to check the action of all involutions in $G_{i}$. In view of $1 \rightarrow\langle\tau\rangle \rightarrow G_{1} \rightarrow \mathbb{Z} / 8 \mathbb{Z} \rightarrow 1$, all involutions of $G_{1}$ lie in the subgroup generated by $\tau$ and $\sigma_{1}^{4}$. Similarly, all involutions of $G_{2}$ lie in the subgroup generated by $\tau$ and $\sigma_{2}^{2}$. It remains to observe that these subgroups are contained in $G$, which is known to act freely [GPo].

Corollary 2.3. For $i=1,2, j=1,2$, the quotients of $V_{8, y}^{i}$ by (the lift of) the group $G_{j}$ are smooth Calabi-Yau threefolds with fundamental groups $G_{j}$ of order 64 .

Proof. By $[\mathrm{GPa}], V_{8, y}^{i}$ are simply connected. It remains to combine Lemma 2.1 and Proposition 2.2.

We remark that $G_{2}$ contains the quaternion group in its regular representation, and $V_{8, y}$ can be thought of as a singular member of the family constructed by 
Beauville in [B]. Also, the quotients $V_{8, y}^{i} / G_{j}$ have Hodge numbers $h^{1,1}=h^{1,2}=2$ and the structures of fibration with abelian surface fibers.

\section{Comments}

We have stumbled upon these examples largely by chance. We originally set out to investigate free actions of finite groups on complete intersections of four quadrics in hopes of extending the construction of [B]. Every such group action naturally leads to a projective representation of dimension eight, which can then be thought of as a linear representation of a Schur cover $S$ of the finite group. If the action is free, the holomorphic Lefschetz formula essentially dictates what character of $S$ one needs to consider, moreover, it leads to strong restrictions on possible group actions. We have used the GAP software package extensively in our search.

It turns out that the maximum possible order of the group (if one allows the variety to have ODP singularities) is 64 . We have found five possible groups of order 64. Upon closer consideration, it turns out that three of them act on the same family of varieties and they are precisely the groups $G, G_{1}$ and $G_{2}$ that appear in this note. The other two, together with the classification of groups of smaller order will be addressed by the second author in $[\mathrm{H}]$.

\section{ACKNOWLEDGMENTS}

We have extensively used the GAP software package in our project, although no computer calculations are necessary to check the results of this paper.

\section{REFERENCES}

[B] A. Beauville, A Calabi-Yau threefold with non-Abelian fundamental group, New Trends in Algebraic Geometry (EuroConference Warwick, July 1996), 13-17. Cambridge University Press (1999). MR1714819 (2000f:14060)

[GPa] M. Gross, S. Pavanelli, A Calabi-Yau threefold with Brauer group $(\mathbb{Z} / 8 \mathbb{Z})^{2}$, preprint math.AG/0512182.

[GPo] M. Gross, S. Popescu, Calabi-Yau threefolds and moduli of abelian surfaces. I, Compositio Math. 127 (2001), 169-228. MR1845899 (2002f:14057)

[H] Z. Hua, in preparation.

Department of Mathematics, University of Wisconsin, Madison, Wisconsin 53706

E-mail address: borisov@math.wisc.edu

Department of Mathematics, University of Wisconsin, Madison, Wisconsin 53706

E-mail address: hua@math.wisc.edu 